\title{
Study of Effect of Quality Nursing Intervention on Negative Emotion and Nursing Job Satisfaction of Patients with Allergic Rhinitis
}

\author{
Xiuhua Shen, Hongwei Zhang, Bing Xu, Jing Wang, Yanhong Yuan
}

Department of Galactophore, Henan Province Hospital of Traditional Chinese Medicine, Zhengzhou 450002

Keywords: quality care intervention; Allergic rhinitis; nursing job satisfaction; quality of life

\begin{abstract}
To investigate the effect of quality nursing intervention on negative emotions and nursing job satisfaction in patients with allergic rhinitis, a total of 167 patients with allergic rhinitis were selected from July 2015 to October 2017 in our hospital. The time of admission was divided into groups. Eighty-three patients in the control group received routine intervention. 84 patients in the study group received high-quality nursing intervention on the basis of the control group. Satisfaction of nursing work was compared between the two groups of depression before and after intervention (SDS), anxiety (SAS score), and quality of life (WHOQOL-100 score). There was no significant difference in SDS and SAS scores between the two groups before intervention $(\mathrm{P}>0.05)$. SDS and SAS scores were lower after intervention in the study group than in the control group $(\mathrm{P}<0.05)$. The total satisfaction rate of the nursing work in the study group was $92.86 \%$ compared with 79.52\% in the control group. High $(\mathrm{P}<0.05)$; WHOQOL-100 score was higher in the study group than in the control group $(\mathrm{P}<0.05)$. Adopting high-quality nursing intervention for patients with allergic rhinitis can significantly relieve negative emotions, improve their quality of life and nursing job satisfaction.
\end{abstract}

\section{Introduction}

Allergic rhinitis is one of the diseases with high incidence in the department of otorhinolaryngology. It is also called allergic rhinitis. It is caused by inhalation of allergens and causes allergic reactions. The incidence is high. According to relevant investigations, allergic rhinitis accounts for approximately all nasal 38\% of the disease [1-2]. The clinical manifestations of many patients were watery nose, nasal itching, nasal congestion, and paroxysmal sneezing, which had adverse effects on their daily work and life [3]. At present, the clinical use of drugs and immunotherapy to treat allergic rhinitis, although to obtain certain results, but the lack of effective care during the treatment of the problem has caused widespread concern in the medical community [4]. High-quality nursing interventions can strengthen guidance and intervention in health knowledge education, mental and emotional education, healthy diet, rational drug use, and functional exercise. It not only reflects the rationality and progress, but also the nursing staff's ideology and medical behavior during the nursing service process. Always centered on patients, it strives to meet its reasonable needs and restores its physical and mental health in the shortest time [5]. In this study, 167 patients with allergic rhinitis were selected to observe the effect of quality nursing intervention on negative emotions and nursing job satisfaction of patients with allergic rhinitis, as follows.

\section{Materials and Methods}

General Information 167 patients with allergic rhinitis were selected from July 2015 to October 2017 in our hospital. The time of admission was divided into groups. The control group consisted of 83 patients, including 34 females, 49 males, aged 21 to 76 years, and average age ( $44.29 \pm 11.51$ ) years old. The study group consisted of 84 patients, including 33 females and 51 males, aged 22 to 77 years and mean age $(45.13 \pm 12.26)$ years. There was no significant difference between the two groups of clinical data $(\mathrm{P}>0.05)$, and this study was approved by the Institute of Ethics of our hospital. 
The control group adopts routine interventions: (1) The nursing staff gives the patient observations and regular dietary interventions; (2) The nursing staff takes regular health education of the patients, including disease common sense, precautions during treatment, etc.; (3) Patients Provides a ward environment with cleanliness and suitable temperature and humidity.

The study group adopts quality care intervention on the basis of the control group: (1) Strengthen psychological enlightenment: determine the psychological needs according to the actual conditions of the patient's education background, work experience, and social growth environment; the nursing staff should actively participate in the treatment The patient communicates, encourages her to complain about her heart, and fully releases her inner depression; nurses can also play soothing, beautiful music and inspirational movies for them to relax and enjoy the next treatment; it can also be used to list allergic rhinitis. Rehabilitation cases have been successful, enhancing their confidence in rehabilitation. (2) Intensified medication guidance: The main drugs for clinical treatment of allergic rhinitis include allergen vaccines, mast cell stabilizers, cholinergic receptor inhibitors, vasoconstrictors, glucocorticoids and antihistamines, and nursing staff. Should be based on their degree of illness, strictly follow the doctor's advice for the selection of suitable drugs, and explain to them the use of drugs and possible adverse reactions, so as to effectively ensure the safety of medication. (3) Intensive diet intervention: Nursing personnel should eat cold food, beef, artificial colors, dairy products, peppers, mustard, and foods containing additives such as eucalyptol, benzaldehyde, vanillin, etc., and eat more parsley, chives, garlic, and ginger. Such warm foods and white radish, Chinese cabbage, Chinese cabbage, spinach and other rich in vitamin foods; at the same time encourage the families of patients to participate in them, together with the patient's daily diet, and then effectively promote their disease recovery. (4) Intensified nasal care: The nursing staff guides them to wash the nasal cavity with physiological saline for 3-5 times a day, making sure that the nasal cavity is hygienic; when flushing the nasal cavity, the physiological saline is poured from the right nostril, the left nostril is under, and the saline is taken from Left nostril outflow, turn the other way to wash the other side of the nasal cavity by the same method; if the weather is cold, rinse the nasal cavity before heating the saline to close to the body temperature and then rinse. (5) Strengthen health education: Through disseminating health handbooks on allergic rhinitis, watching videos, and conducting weekly special lectures to promote health knowledge of allergic rhinitis, including disease mechanisms, major allergens, clinical manifestations, and treatment methods. Common complications and other common sense; focus on inhalation of pollen, dander, dust mite and other allergens and flour, peanuts, fish and shrimp and other food allergens, and teach them to avoid allergens methods and techniques. (6) Strengthen the living environment care: regularly clean the pillowcases, duvet covers, bed sheets and clean air-conditioning filters. The wards often open the windows for ventilation; pay attention to keep warm, beware of cold stimulation to induce the onset of the disease; give necessary exercise guidance to improve the patient's resistance; Smoking, avoiding use of carpets, prohibiting pets from entering the bedroom, and not putting flowers in the room; use less repellents, perfumes, and other sensitive chemicals.

Observing indicators (1) Before and after the intervention, depression and anxiety self-assessment scales (SDS, SAS) were used to evaluate the negative emotions of the two groups. The higher the score, the more severe the negative emotions. (2) Satisfy the satisfaction of two groups with a self-made nursing satisfaction score table, with scores of $<74$ being unsatisfactory; scores of 74-89 being rated as satisfactory; scores of $>89$ being very satisfied, and being satisfied, very satisfied being included in the total satisfaction rate . (3) Before and after the intervention, the WHO quality of life scale (WHOQOL-100) was used to evaluate the quality of life of the two groups. The lower the score, the worse the quality of life.

1.4 Statistical analysis SPSS 23.0 processing, measurement data $( \pm \mathrm{s}), \mathrm{t}$ test, count data to $\mathrm{n}(\%)$, chi square test, test standard $\alpha=0.05$.

\section{Results}

SDS and SAS scores The scores of SDS and SAS before the intervention were not significantly different $(\mathrm{P}>0.05)$. The SDS and SAS scores of the study group after intervention were lower than 
those of the control group $(\mathrm{P}<0.05)$. See Table 1 .

Table 1 SDS, SAS scores ( \pm s, points)

\begin{tabular}{|c|c|c|c|c|c|}
\hline \multirow{2}{*}{ Gr } & \multirow{2}{*}{$\mathrm{n}$} & SDS & \multicolumn{3}{|c|}{ SAS } \\
\hline & & Before & After & Before & After \\
\hline research group & 84 & $63.51 \pm 5.96$ & $40.67 \pm 3.08$ & $60.42 \pm 3.38$ & $39.48 \pm 3.27$ \\
\hline Control group & 83 & $62.87 \pm 6.53$ & $45.89 \pm 4.63$ & $59.73 \pm 3.75$ & $43.15 \pm 4.32$ \\
\hline$t$ & 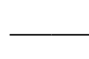 & 0.328 & 6.249 & 0.627 & 6.048 \\
\hline$P$ & 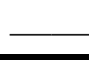 & 0.751 & 0.000 & 0.416 & 0.000 \\
\hline
\end{tabular}

The total satisfaction rate of $92.86 \%$ in the nursing work of the study group was higher than that of the control group $(79.52 \%)(\mathrm{P}<0.05)$. See Table 2 .

Table 2 Nursing Job Satisfaction n (\%)

\begin{tabular}{lccccc}
\hline Gr & $\mathrm{n}$ & Not satisfied & satisfied & $\begin{array}{l}\text { Very } \\
\text { satisfied }\end{array}$ & $\begin{array}{l}\text { Total } \\
\text { satisfaction rate }\end{array}$ \\
\hline $\begin{array}{l}\text { research } \\
\text { group }\end{array}$ & 84 & $6(7.14)$ & $43(51.19)$ & $35(41.67)$ & $78(92.86)$ \\
$\begin{array}{l}\text { Control } \\
\text { group }\end{array}$ & 83 & $17(20.48)$ & $44(53.01)$ & $22(26.51)$ & $66(79.52)$ \\
$\chi^{2}$ & - & - & - & - & 6.255 \\
$P$ & - & - & - & - & 0.012 \\
\hline
\end{tabular}

There was no significant difference between the WHOQOL-100 score $(59.24 \pm 8.89)$ and the control group (58.73 \pm 9.24$)$ before the intervention $(\mathrm{t}=0.754, \mathrm{P}=0.380)$. WHOQOL-100 score (91.26 \pm 6.87$)$ points after the study group intervention. It was higher than the control group $(85.34 \pm 5.29)(t=4.685, \mathrm{P}=0.000)$.

\section{Discussion}

Allergic rhinitis refers to the chronic inflammatory diseases of the nasal mucosa that are produced by the individual's own immune system after exposure of specific individuals to allergens, including both perennial and seasonal types [6]. In recent years, the incidence of allergic rhinitis has increased significantly worldwide, and it has become one of the thorny issues facing the world [7]. Allergic rhinitis is difficult to cure, and the recurrence rate is high, which greatly affects the daily life of patients, combined with a long course of disease, it also brings a greater mental and economic burden, resulting in a decrease in treatment compliance, and a certain degree of effect on the treatment [ 8]. Therefore, in the treatment of patients with allergic rhinitis, it is of great significance to implement corresponding nursing interventions.

In the past, clinical interventions were often used, but due to the lack of targeted nursing measures and high-quality nursing services, the patients' enthusiasm for treatment was not effectively improved and the overall effect was poor. High-quality nursing intervention model has become increasingly prominent in the nursing field in recent years. Its service concept is to emphasize basic care and provide individuals with satisfactory services[9]. Scholar Feng Fen[10] studies have shown that patients undergoing laparoscopic cholecystectomy with high-quality nursing intervention can significantly reduce their bad mood, shorten bowel sound recovery time, discharge time and defecation time, and help improve their postoperative Quality of Life. This study shows that the SDS and SAS scores were lower in the study group than in the control group $(\mathrm{P}<0.05)$. It can be seen that adopting quality nursing interventions for patients with allergic rhinitis can significantly relieve negative emotions. The analysis may be due to: quality nursing intervention can strengthen psychological guidance, effectively resolve negative emotions of 
patients and improve their psychological state of mind, not only to increase their enthusiasm for treatment, but also help establish a good nurse-patient relationship.

This study will apply quality care interventions to patients with allergic rhinitis, can significantly enhance daily care, provide better, more complete, and more targeted care services, and at the same time improve the quality of care services, to ensure the safety of care measures, Effective, and thus greatly enhance the patient's satisfaction with nursing work. From this study, we found that the total satisfaction rate of nursing work in the study group was $92.86 \%$ higher than that of the control group $(79.52 \%)(\mathrm{P}<0.05)$, and the WHOQOL-100 score was higher in the study group than in the control group $(\mathrm{P}<0.05)$. It shows that adopting high-quality nursing intervention for patients with allergic rhinitis can significantly increase their satisfaction with nursing work and quality of life. The reason is that high-quality nursing interventions can strengthen awareness of disease knowledge and enable patients to understand the major allergens causing the disease, and at the same time master the methods and techniques to avoid such allergens, thereby effectively reducing the frequency of allergic rhinitis; it can also choose the right one based on the patient's actual condition. Drugs, and strict compliance with the doctor's advice, so as to effectively ensure the efficacy of treatment and medication safety; also strengthen daily care and dietary interventions to ensure that patients fully ingest the necessary nutrients, which will help patients recover soon and promote their quality of life improvement [11].

In summary, adopting high-quality nursing intervention for allergic rhinitis patients can significantly ease their negative emotions, and improve their quality of life and job satisfaction.

\section{References}

[1] Yan Yan, Li Peng, Liu Dandan. The efficacy of montelukast sodium, budesonide nasal spray, and loratadine in combination in the treatment of allergic rhinitis and its influence on inflammatory factors [J]. Chinese Pharmacist, 2016, 19(10): 1900-1901, 1921.

[2] Wang Renxia, Zhang Chunhui. Clinical efficacy analysis of montelukast sodium combined with budesonide nasal spray and loratadine in the treatment of moderate-severe persistent allergic rhinitis [J]. Clinical Otolaryngology Head and Neck Surgery Magazine, 2015, 29(23): 2041-2043.

[3] FU Kun, LIU Haibing, LI Xiaoming, et al. Mechanism of action of loratadine combined with budesonide in the treatment of allergic rhinitis[J]. Southwest China Medical and Pharmaceutical Classification, 2016, 26(3): 305-307.

[4] Xin Xu. Nursing intervention for specific immunotherapy of allergic rhinitis[J]. Journal of clinical nursing,2013,12(4):35-37.

[5] Xue Xiaoling, Wan Runqin. Effect of quality nursing intervention on gastrointestinal function and VAS score after laparoscopic cholecystectomy[J]. Journal of Clinical Medicine in Practice,2017,21(16):65-68.

[6] Liao Ming, Li Yong, Tao Baohong. Effects of loratadine on peripheral blood $\mathrm{T}$ cells 17 in children with allergic rhinitis[J]. Chinese Journal of Clinical Pharmacology, 2015, 31(14):1366-1368.

[7] Hou Mingyue. Comparison of the efficacy of loratadine and montelukast sodium in the treatment of seasonal allergic rhinitis[J]. Shandong Medical Journal,2016,56(26):99-101.

[8] Li Xiaomin, Hong Haiyu, Guo Jianling, et al. Long-term efficacy evaluation of specific immunotherapy for allergic rhinitis and observation of efficacy of prophylactic use of loratadine[J]. Guangdong Medical Science, 2014,35(2):282- 284.

[9] Cao Qian, Fang Na, Liu Qiaofen. Observation of the effect of clinical quality nursing model in patients with cholecystitis[J]. Hebei Medical Journal, 2017, 39(23): 3668-3670.

[10] Feng Fen. Efficacy observation and nursing experience of laparoscopic cholecystectomy with quality nursing[J]. Journal of Clinical Medicine in Practice,2016,20(14):86-88.

[11] Zhou Meirong. Study on the role of different nursing methods in promoting the efficacy of allergic rhinitis $[\mathrm{J}]$. Journal of Otorhinolaryngology, Chinese Journal of Integrated Traditional and Western Medicine, 2017, 25(4):313-315. 